\title{
RECONSTRUCTION OF THE INTERNAL JUGULAR VEIN BY FEMORAL VEIN SEGMENT INSERTION IN A PATIENT AFTER BILATERAL NECK DISSECTION
}

\author{
Kinga Jupowicz-Marciniak', Bogdan Kolebacz'1, Grażyna Stryjewska-Makuch'1, \\ Piotr Piesik ${ }^{2}$
}

'Department of Otolaryngology and Laryngological Oncology of the Independent Public Research Hospital No. 7 of the Medical University of Silesia in Katowice, Poland ${ }^{2}$ Department of General Surgery, Vascular Surgery, Angiology, and Phlebology of the Clinical Hospital No. 7 of the Medical University of Silesia in Katowice, Poland

\begin{abstract}
In this paper we describe a patient who underwent radical neck dissection with bilateral excision of the internal jugular veins because of recurrent laryngeal cancer with infiltration of both internal jugular veins. One of the jugular veins was reconstructed using a segment of the femoral vein. Follow-up 12 months after the procedure revealed normal flow through the reconstructed vein, no clinical signs of impaired venous outflow from the head and neck, and no leg oedema, despite lacking a segment of the femoral vein. In selected patients presenting with advanced cancers of the neck, with other veins, such as the external jugular vein or saphenous vein, not suitable for use as a graft, this method of reconstruction of the internal jugular vein can be performed in order to protect the patient from complications associated with impaired venous outflow.
\end{abstract}

Key words: internal jugular vein, vascular reconstruction, femoral vein.

\author{
CASE REPORT \\ Phlebological Review 2016; 24, 4: 71-74 \\ D0l: https://doi.org/10.5114/pr.2016.67745
}

Submitted: 11.11 .2016

Accepted: 21.02.2017

\section{ADDRESS FOR CORRESPONDENCE}

Kinga Jupowicz-Marciniak

Department of Otolaryngology and

Laryngological Oncology of the Independent

Public Research Hospital No. 7 of the Medical

University of Silesia in Katowice

Ziolowa $45-47$

40-635 Katowice, Poland

e-mail: kinga.jupowicz@gmail.com

\section{INTRODUCTION}

Laryngeal carcinoma (LC) is the most common cancer of the head and neck. Squamous cell cancers account for $95 \%$ of malignant tumours of the larynx [1]. LC remains a significant medical and social problem, in spite of progress in the diagnosis and treatment of this disease. Failures in treatment for LC primarily result from relapses to the lymphatic system of the neck. Squamous cell cancers located in the glottis usually are highly or moderately differentiated (G I-II). Poorly differentiated tumours (G III) are primarily found in the upper part of the larynx. These cancers exhibit fast growth and metastasise early to the lymph nodes, with the incidence of such metastases at the level of 50-60\% [1]. Cancers of the middle part of the larynx exhibit slow progression and rarely metastasise to the lymph nodes, with incidence of such metastases at the level of only $4-10 \%$ [1]. This phenomenon is due to a sparse lymphatic network in this area and usually highly differentiated characteristics of these tumours. A subglottic cancer is typically characterised by moderate growth, and metastases occur in $10-20 \%$ of these patients [1]. Depending on the location of the tumour, metastases are found in different groups of the cervical lymph nodes. Metastases to the lymph nodes of the lower part of the neck (group IV and VB) may originate in the larynx, pharynx, and thyroid gland, and also in the organs located outside the head and neck [2]. Primary laryngeal tumours as well as metastatic lymph nodes of group III-V can infiltrate large vessels of the neck, the internal jugular vein in particular. In such an advanced neoplastic disease the surgeon is often challenged by a difficult decision to remove both the lymphatic and the venous systems on either side of the neck. Consequently, venous outflow from the brain can be compromised and reconstruction of the jugular vein can be necessary. In this paper we describe a patient who underwent bilateral removal of the lymphatic system of the neck and also bilateral excision of the internal jugular veins. One of the jugular veins was reconstructed using a segment of the femoral vein.

\section{CASE REPORT}

In January 2013 a 57-year-old male patient was admitted to the Department of Otolaryngology of our hospital because of a three-month history of hoarseness. Laryngological examination revealed an exophytic tumour located along the anterior and middle part of the right vocal fold. A biopsy was taken from this lesion; histopathological examination found planoepithelial carcinoma, stage 
G2. In February 2013 we performed partial laryngectomy, with excision of the right vocal fold, anterior commissure, and adjacent tissues. Histopathological examination of resected right vocal fold revealed carcinoma planoepitheliale akeratodes G2. Margins were free of neoplastic infiltration. At follow-up visit in July 2013 laryngological examination demonstrated a fragile bleeding infiltration of the left vocal fold, which was localised along its anterior part and descended to the subglottic area. The left vocal fold was immobile. A biopsy taken from this lesion confirmed the presence of a neoplasm. In July 2013 we performed total laryngectomy. Preoperative diagnostics did not demonstrate enlarged lymph nodes. Nevertheless, intraoperatively was found an enlarged pre-laryngeal lymph node, which was also excised. Histopathology of this lymph node showed reactive lymphadenitis. In June 2014 the patient was again admitted to our department because of bilateral enlargement of the cervical lymph nodes. Computed tomography scans performed at that time (Figs. 1 and 2) showed a conglomerate of enlarged lymph nodes to the right of the thyroid gland and behind the sternocleidomastoid muscle (diameters: $58 \times 43 \times 55$ $\mathrm{mm}$ ). One of these lymph nodes, located most laterally, was the largest one (30 $\mathrm{mm}$ in diameter) and hypodense, probably due to a regressive process. All these enlarged lymph nodes directly adhered to a 4 - $\mathrm{cm}$ segment of the internal jugular vein. The vein was also compressed by this infiltration.

In addition, one of the enlarged lymph nodes adhered to a $1.2-\mathrm{cm}$ segment of the internal carotid artery. On the left side, laterally from the thyroid gland, there was a smaller conglomerate of lymph nodes (diameters: 31 $\times 26 \times 38 \mathrm{~mm}$ ). We planned to perform modified radi-

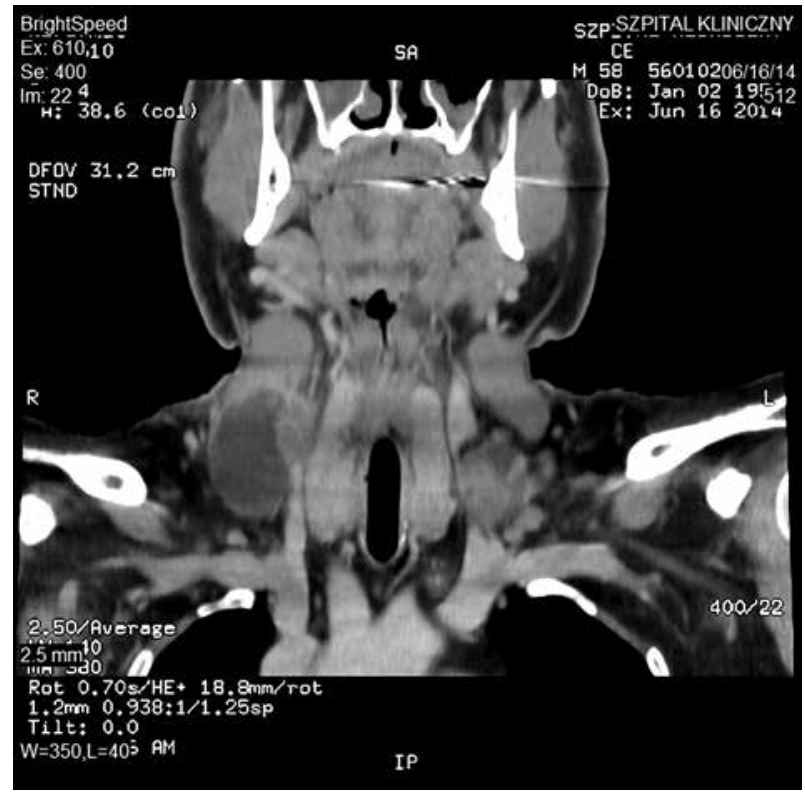

Fig. 1. Contrast-enhanced computed tomography of the neck. Visible lymph node packages adjacent to the jugular veins cal neck dissection (MRND) on the right side together with excision of conglomerate of the lymph nodes on the left side. Nonetheless, intraoperatively neoplastic disease appeared to be more advanced than expected. Enlarged lymph nodes on the right side belonged to groups IV and V, while on the left side to groups III, IV, and V. These lymph nodes infiltrated the left internal jugular vein. Because before the surgery the patient did not consent for bilateral excision of the internal jugular veins, we completed the operation. The patient was then consulted by a vascular surgeon who, in order to restore blood outflow from the brain, suggested neck dissection with simultaneous reconstruction of the jugular veins. The patient underwent sonographic assessment of the veins of lower extremities. This examination demonstrated patent superficial and deep veins of both lower extremities. Still, on both sides the great saphenous veins were of small diameter and thus unsuitable as reconstruction material.

Therefore, we decided to use a segment of femoral vein to reconstruct the jugular veins. About one month after the previous exploratory operation, under general anaesthesia, we removed bilaterally lymph node packages extending from the level of bulb of the common carotid artery to the level of clavicle. In addition to the lymph nodes, also the accessory nerves, the adipose tissue of the supraclavicular fossa, and both internal jugular veins were resected. Then, the vascular reconstruction of the left internal jugular vein was performed. For this purpose, we exposed the left femoral vein and harvested a $10-\mathrm{cm}$-long segment located distally from the deep femoral vein. We used this vein to reconstruct the resected vein, using end-to-end anastomoses with a continuous 5.0 Prolene suture. The patient was discharged with the

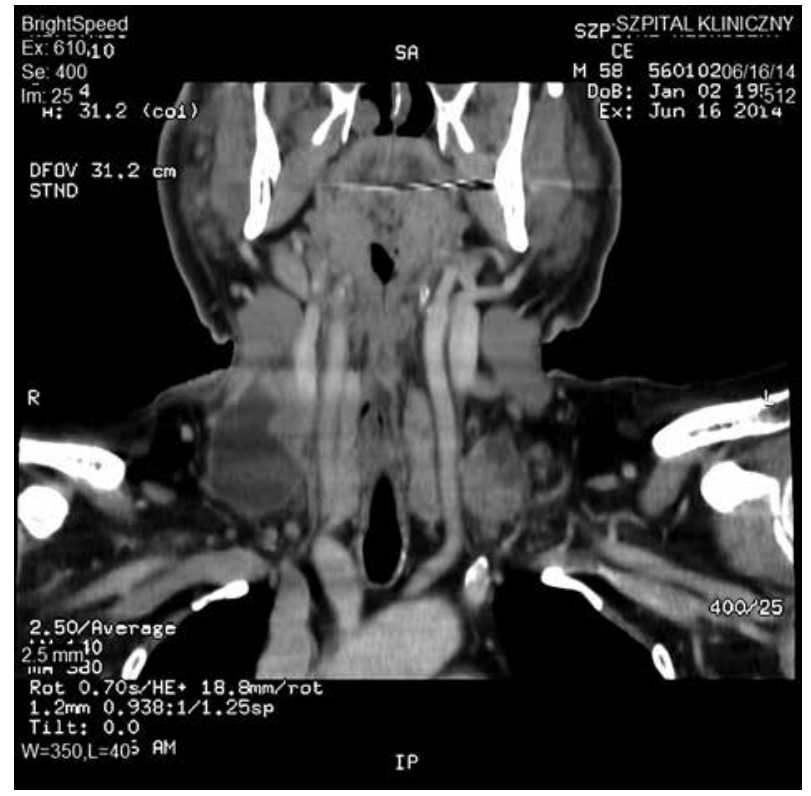

Fig. 2. Contrast-enhanced CT of the neck. Visible lymph node packages relative to jugular veins 


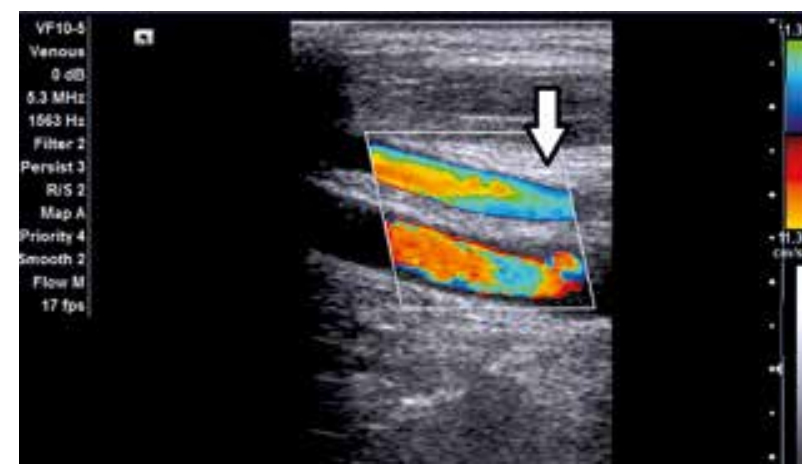

Fig. 3. Colour-coded Doppler sonography of the carotid artery and transplanted femoral vein (arrow) -6 months after surgery

recommendation of an anticoagulation with low-molecular-weight heparin for four weeks, and antiplatelet therapy with aspirin $150 \mathrm{mg}$ daily. There was normal flow through the reconstructed jugular vein, demonstrated by Doppler sonography at the patient's discharge from the hospital. Compression therapy of the left lower extremity, initially with the use of elastic bandages and then with a class 2 compression stocking, was also advised. After oncological consultation the patient received chemoradiotherapy. He remains under constant laryngological and vascular follow-up. Doppler sonography performed three, six, and 12 months after the surgery confirmed patency of reconstructed venous segment, without sonographic signs of thrombosis (Figs. 3 and 4). Also, the patient did not complain of leg oedema, and sonographic assessment of veins of the lower extremities did not reveal venous thrombosis. The patient is, at the time of writing, wearing the recommended compression stockings and he is free from cancer recurrence.

\section{DISCUSSION}

Radical dissection of the lymphatic system of the neck was described for the first time in the year 1888 by the Polish surgeon Franciszek Jawdyński. In 1906 this procedure was also reported by Crile as a "comprochense neck dissection" [3]. Since the 1960s the details of this procedure have been gradually amended by many surgeons. Currently, neck dissections comprise several types of surgical procedures:

- the Jawdyński-Crile procedure, which involves the removal of all five groups of the cervical lymph nodes: submental and submandibular lymph nodes, lymph nodes along the internal jugular vein, lymph nodes of the posterior triangle of the neck, and other anatomical structures of the neck, such as the sternocleidomastoid muscle, the accessory nerve, and the internal jugular vein;

- modified procedure, which comprises resection of all groups of the lymph node (I-V) with preservation of

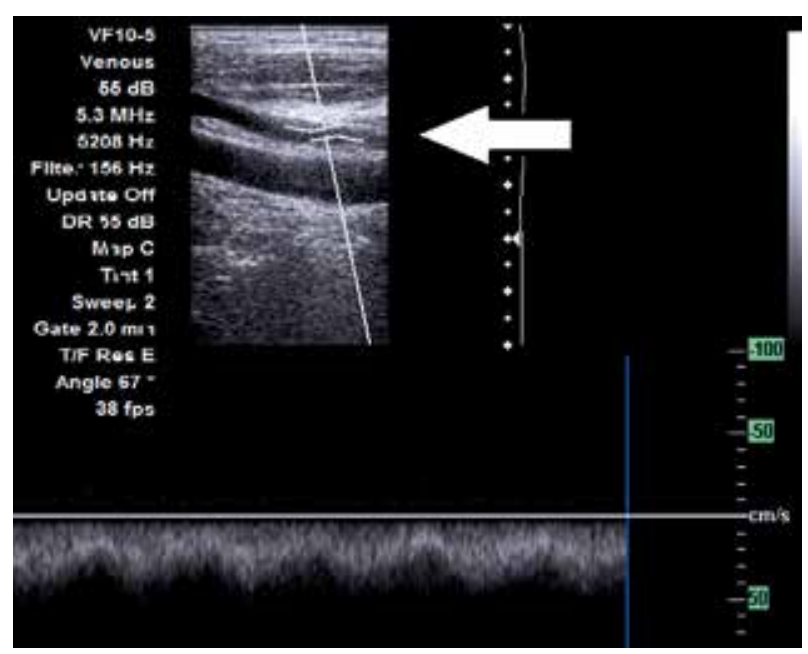

Fig. 4. Duplex Doppler sonography or the transplanted vein (arrow) - 6 months after surgery

one or more of the above-mentioned non-lymphatic structures of the neck;

- effective (preventive) procedure, consisting of removal of enlarged cervical lymph nodes with no obvious signs of malignancy but presenting with high risk of an occult metastasis [3].

Bilateral radical neck dissection is carried out as an elective procedure, simultaneously with the excision of neoplastic tumour, or as a secondary operation performed because of metastases to the contralateral cervical lymph nodes. A one-step radical neck dissection was described in the 1950s [4]. Since then a number of publications [5-8] have confirmed safety of such a dissection. In order to develop a better venous collateral circulation some authors suggested performing the surgery in two steps, with a 2-4-week interval between the procedures [9, 10]. Crile himself, who in selected patients recommended bilateral excision of the internal jugular veins, claimed that the deep cervical and vertebral veins would enable a proper cerebral venous outflow. The vertebral and deep cervical veins carry venous blood from the suboccipital venous plexus. The deep cervical veins exhibit numerous connections with the external vertebral plexus and veins draining the muscles of the neck. Usually they join the vertebral vein and make a common venous trunk, which below the level of the transverse process of the seventh cervical vertebra drain into the brachiocephalic vein, and less often into the subclavian vein. The occipital sinus and the basilar plexus join the internal vertebral plexuses, constituting another important outflow route after a ligation of the internal jugular vein.

Studies on these alternative venous flow routes were presented in Ensari's paper [10]. Unfortunately, there were also reports on severe complications following bilateral ligation of the internal jugular veins, such as facial or throat oedema, visual impairment or even blindness, thrombosis of the intracranial veins, and fatal outcomes 
[7, 11-13]. These adverse events encouraged surgeons to reconstruct the internal jugular vein, using as a graft the posterior or external jugular veins, or the great saphenous vein, with an end-to-end or end-to-side anastomosis and adjusting the diameter of transplanted blood vessels to the desired diameter of the reconstructed jugular vein. Reconstruction with polytetrafluoroethylene prosthesis was also an option $[14,15]$.

Katsuno [16] proposed three types of internal jugular vein reconstructions. In type $A$, he connected, using the end-to-end anastomosis, the internal jugular vein with the external jugular vein. In type $B$, the internal jugular vein was reconstructed using the saphenous vein interposition graft connected with the stumps of resected internal jugular vein, and in type $C$ the inserted segment was anastomosed cranially with the internal jugular vein, and caudally with the external jugular vein. Kamizono [9] proposed the new type $\mathrm{K}$, connecting the internal jugular vein with the anterior jugular vein using end-to-end anastomosis. The prerequisite of this method is to preserve the anterior jugular vein of a proper diameter, which the authors propose to assess preoperatively by means of Doppler sonography or contrast-enhanced CT scanning. Careful preservation of the facial and retromandibular veins and type $\mathrm{K}$ reconstruction of the internal jugular vein protect the patient from facial and neck oedema [9]. In our patient we performed type B reconstruction, with the use of femoral vein instead of a saphenous vein because the external and anterior jugular veins were excised during the operation and the saphenous vein was too small to be used as a graft. We did not observe postoperative facial or neck oedema, probably because of adequate collateral venous network.

\section{CONCLUSIONS}

Despite generally poor prognosis in patients presenting with advanced neoplastic disease of the neck, a decision to perform reconstruction of the internal jugular vein after bilateral radical neck dissection can protect patients from complications associated with impaired venous outflow.

\section{The authors declare no conflict of interest.}

\section{References}

1. Osuch-Wójcikiewicz E. Otolaryngologia praktyczna. Podręcznik dla studentów i lekarzy. G. Janczewski (ed.). Via Medica Gdańsk 2007; pp. 507-514.

2. Nowotwory nabłonkowe narządów głowy i szyi. Kawecki A., Nawrocki S. (eds.), Via Medica Gdańsk 2013.

3. Waśniewska-Okupniak E., Wierzbicka M., Szyfter W. Nowotwory w otolaryngologii. Szyfter W. (ed.). Termedia, Poznań 2015; 94-99.

4. Gius J.A., Grier D.H. Venous adaptation following bilateral radical neck dissection with excision of the jugular veins. Surgery 1950; 28: 305-321.
5. Frazell E.L., Moore O.S. Bilateral radical neck dissection performed in stages. Experience with 467 patients. Am J Surg 1961; 102: 809-814.

6. McGuirt W.F., McCabe B.F. Bilateral radical neck dissections. Arch Otolaryngol 1980; 106: 427-429.

7. Razack M.S., Baffi R., Sako K. Bilateral radical neck dissection. Cancer 1981; 47: 197-199.

8. Moore O.S., Frazell E.L. Simultaneous bilateral neck dissection: Experience With 151 Patients. Am J Surg 1964; 107: 565-568.

9. Kamizono M., Ejima M., Taura M., Masuda M. Internal jugular vein reconstruction: application of conventional type $\mathrm{A}$ and novel type K methods. J Laryngol Otol 2011; 125: 643-648.

10. Ensari S., Kaptanoğlu E., Tun K., Gün T., Beşkonakli E., Celikkanat S., Dere H., Cekirge S. Venous outflow of the brain after bilateral complete jugular ligation. Turk Neurosurg 2008; 18 : 56-60.

11. Frazell E.L., Moore O.S. Bilateral radical neck dissection performed in stages. Experience with 467 patients. Am J Surg 1961; 102: 809-814.

12. Eibling D.E., Snyderman C.H., Weber P.C., Johnson J.T., Steed D.L. Internal jugular vein reconstruction in bilateral radical neck dissection. Am J Otolaryngol 1995; 16: 260-264.

13. Dulguerov P., Soulier C., Maurice J., Faidutti B., Allal A.S., Lehmann W. Bilateral radical neck dissection with unilateral internal jugular vein reconstruction. Laryngoscope 1998; 108: 1692-1696.

14. Dua A., Desai S.S. Utility of internal jugular vein reconstruction in modified radical neck dissection. Vascular 2014; 22: 81-84.

15. Zhenhu R., Tengfei F., Hanjiang W., Kai W., Hongyu T., Chaojian G., Liu J. Jugular vein reconstruction by longitudinal constriction suture venoplasty and microvascular anastomosis. Hua Xi Kou Qiang Yi Xue Za Zhi 2014; 32: 476-479.

16. Katsuno S., Ishiyama T., Nezu K., Usami S. Three types of internal jugular vein reconstruction in bilateral radical neck dissection. Laryngoscope 2000; 110: 1578-1580. 